\title{
In vivo functional photoacoustic tomography of traumatic brain injury in rats
}

Jung-Taek Oh, Kwang-Hyun Song, Meng-Lin Li, George Stoica, Lihong V. Wang

Jung-Taek Oh, Kwang-Hyun Song, Meng-Lin Li, George Stoica, Lihong V. Wang, "In vivo functional photoacoustic tomography of traumatic brain injury in rats," Proc. SPIE 6086, Photons Plus Ultrasound: Imaging and Sensing 2006: The Seventh Conference on Biomedical Thermoacoustics, Optoacoustics, and Acousto-optics, 60860P (6 March 2006); doi: 10.1117/12.645378

SPIE. Event: SPIE BiOS, 2006, San Jose, California, United States 


\title{
In vivo functional photoacoustic tomography of traumatic brain injury in rats
}

\author{
Jung-Taek Oh, Kwang-Hyun Song, Meng-Lin Li \\ Optical Imaging Laboratory, Department of Biomedical Engineering, Texas A\&M University, TX \\ 77843-3120 \\ George Stoica \\ Department of Pathobiology \\ Texas A\&M University, College Station, TX 77843-5547 \\ Lihong V. Wang* \\ Optical Imaging Laboratory, Department of Biomedical Engineering, Texas A\&M University, TX \\ $77843-3120$
}

\begin{abstract}
In this study, we demonstrate the potential of photoacoustic tomography for the study of traumatic brain injury (TBI) in rats in vivo. Based on spectroscopic photoacoustic tomography that can detect the absorption rates of oxy- and deoxyhemoglobins, the blood oxygen saturation and total blood volume in TBI rat brains were visualized. Reproducible cerebral trauma was induced using a fluid percussion TBI device. The time courses of the hemodynamic response following the trauma initiation were imaged with multi-wavelength photoacoustic tomography with bandwidth-limited spatial resolution through the intact skin and skull. In the pilot set of experiments, trauma induced hematomas and blood oxygen saturation level changes were detected, a finding consistent with the known physiological responses to TBI. This new imaging method will be useful for future studies on TBI-related metabolic activities and the effects of therapeutic agents.
\end{abstract}

Keywords: photoacoustic tomography, traumatic brain injury, spectroscopic imaging, blood oxygenation, blood volume

\section{INTRODUCTION}

Despite the development of neurochemical, histopathological and molecular techniques for studying traumatic brain injury (TBI), no neuroprotective therapy is currently available, and TBI remains one of the leading causes of disability and death in young adults in industrialized countries. ${ }^{1}$ TBI can be categorized into focal and diffuse injuries to the brain. Focal injury is characterized by the presence of contusions, which are often accompanied by hematomas. In the case of diffuse injuries, diffuse brain swelling and ischemic brain damage injury are observed not only in the direct vicinity but also remotely from the site of impact. ${ }^{2}$ Currently, the primal goal of the management of traumatic brain injury is the prevention of secondary damage due to neuronal hypoxia and hypoperfusion. Most of the imaging and monitoring modalities are aimed at identifying potential episodes of hypoxia and guiding therapy related to cerebral perfusion. ${ }^{3}$

So far, several technologies have been explored as noninvasive TBI imaging methods. Positron emission tomography (PET) and single photon emission tomography (SPECT) provide cerebral flow and volume information with exogenous contrast agents, but their spatial resolutions are on the order of $\mathrm{cm}$, which is the size of only very small brains. ${ }^{4} \mathrm{fMRI}$ provides excellent details about cerebral perfusion, and it is used to pickup dynamic changes in deoxy-hemoglobin. However, a quantitative oxygen saturation value can't be extracted because of its insensitivity to oxy-hemoglobin. Also,

\footnotetext{
* Corresponding author: LWang@tamu.edu.
} 
the image resolution is not satisfactory for imaging blood vessels in small animal brains. ${ }^{5}$ Diffuse optic tomography can also be used to detect oxygen saturation based on the intrinsic absorption spectrum of hemoglobin. ${ }^{6}$ However, its resolution and accuracy are limited by light scattering in the biological tissues when imaging the brain through the intact skin and skull.

Functional photoacoustic tomography (fPAT) is a new non-invasive imaging modality, and it is emerging as a very practical method for imaging biological tissue structures by means of laser-induced ultrasound. ${ }^{7}$ Structures with high optical absorption, such as blood vessels, can be imaged with the spatial resolution of ultrasound, which is not limited by the strong light scattering in biological tissues. By varying the wavelengths of the laser light and acquiring photoacoustic images, the optical absorption spectrum of each image pixel can be found. Since the biochemical constituents of tissues determine the spectrum, useful functional information, like oxygen saturation $\left(\mathrm{SO}_{2}\right)$ and total haemoglobin concentration $(\mathrm{HbT})$, can be extracted. The level of oxygen saturation of the vasculature of the brain cortex has been successfully measured by functional photoacoustic tomography. ${ }^{8}$

In this study, we demonstrate the application of functional photoacoustic tomography to the study of traumatic brain injury (TBI) in rats in vivo. High resolution brain cortex vasculature images of oxygen saturation and total hemoglobin concentration are produced to visualize hemodynamic changes prior to and following the TBI. Because of the location of the injury site inside the brain, conventional optical imaging modalities have so far been unable to detect in vivo homodynamic information at the level of sub $\mathrm{mm}$ spatial resolution. The commonly used fluid percussion model was employed to mimic TBI in small animals, and hemorrhaging from the injury was identified by the low oxygen saturation $\left(\mathrm{SO}_{2}\right)$ and high total hemoglobin concentration $(\mathrm{HbT})$ of the vasculature. Differential images of $\mathrm{SO}_{2}$ and $\mathrm{HbT}$ before and after the TBI were acquired to visualize the physiological changes. In addition to imaging the whole brain, one position of the brain was monitored continuously using a photoacoustic signal every 1 minute to pick up the transient hemodynamics caused by the TBI.

\section{Material and methods}

\subsection{Experimental setup}

The experimental setup for the in vivo functional photoacoustic tomography of a brain tumor in-vivo is shown in Fig. 1. A tuneable Ti:Sa nanosecond pulse laser (LT-2211A, Lotis T II, Minsk, Belarus) pumped by an Nd:YAG laser (LS2137/2, Lotis T II, Minsk, Belarus) was employed to provide laser pulses with a pulse repetition rate of $10 \mathrm{~Hz}$. The laser beam was expanded by a light diffuser and then delivered to the animal head. The incident energy density of the laser beam on the surface of the mouse head was controlled at $\sim 30 \mathrm{~mJ} / \mathrm{cm}^{2}$ (within the ANSI standard). Laser pulse energy was measured with a photodiode, and the measured energy was used to compensate for fluctuations in the laser output and corresponding photoacoustic signals. Ultrasonic transducers (V323/2.25MHz, Panametrics, $50 \%$ bandwidth) were used as detectors to receive the induced acoustic signals. The large active area $(6 \mathrm{~mm}$ diameter $)$ of the transducer increased the sensitivity. Four wavelengths, $764 \mathrm{~nm}, 784 \mathrm{~nm}, 804 \mathrm{~nm}$ and $824 \mathrm{~nm}$, were adopted in each scanning step. A step motor drove the transducers to circularly scan with a step size of 3 degrees. The mouse was held by a homemade fixture with its head protruding into the water tank through a hole in the bottom. The hole was sealed with a piece of thin plastic film. The mouse head surface was covered with a thin layer of ultrasonic coupling gel. The detected photoacoustic signals were amplified and then digitized by a Gage PC card (CS14100, Gage). The distribution of the optical absorption in the imaging plane (x-y plane) was reconstructed using a modified back-projection algorithm after a full view scanning. ${ }^{9}$ The measured spatial resolution was $0.26 \mathrm{~mm}$ from the FWHM of the point spread function.

\subsection{Theory}

Images from four wavelengths of the light source were processed to create $\mathrm{HbT}$ and $\mathrm{SO}_{2}$ maps. The method for determining both the $\mathrm{HbT}$ and the $\mathrm{SO}_{2}$ in the vasculature from the photoacoustic image information was based on that of Xuiding et al. using the following model: ${ }^{8}$

$$
P A_{\lambda}=k \varepsilon_{\lambda}^{H b}[\mathrm{Hb}]+k \varepsilon_{\lambda}^{\mathrm{HbO}_{2}}\left[\mathrm{HbO}_{2}\right]
$$


Wave length tuning $(\lambda: 764,784,804,824 \mathrm{~nm})$

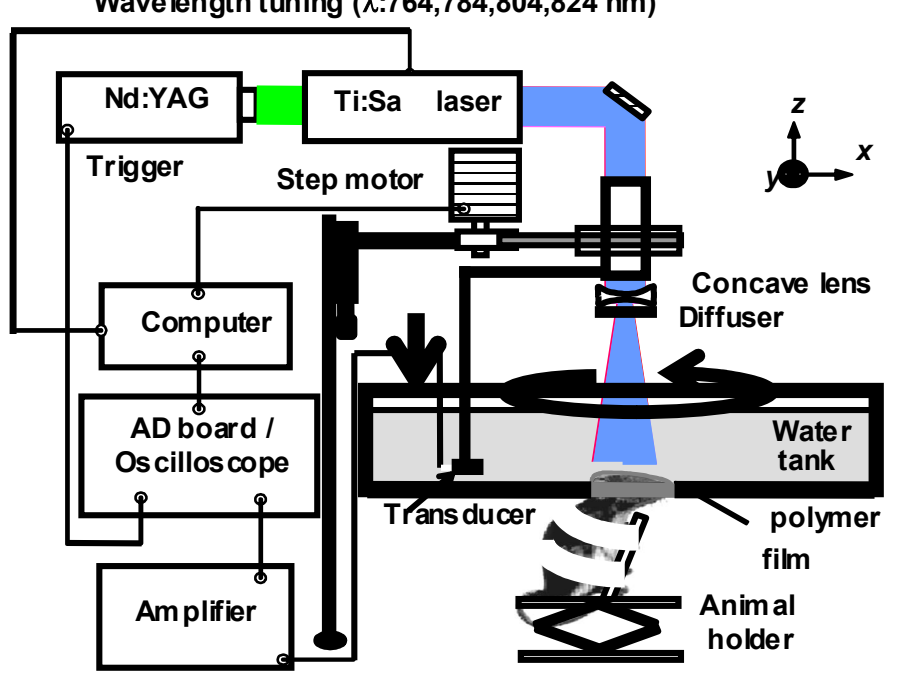

Figure 1. Schematic of the functional PAT system for functional imaging of the hemoglobin concentration and oxygenation in a rat brain in vivo.

$P A_{\lambda}$ is the pixel value of reconstructed photoacoustic image at the wavelength $\lambda ; \varepsilon_{\lambda}{ }^{\mathrm{Hb}}$ and $\varepsilon_{\lambda}{ }^{\mathrm{HbO} 2}$ are the extinction coefficients for deoxy-haemoglobin $(\mathrm{Hb})$ and oxy-haemoglobin $\left(\mathrm{HbO}_{2}\right)$ for wavelength $\lambda$, respectively; [Hb] and $\left[\mathrm{HbO}_{2}\right]$ are the concentrations of deoxy- and oxy-hemoglobin, repectively; and $k$ is the scaling constant. In this model, it is assumed that $\mathrm{Hb}$ and $\mathrm{HbO} 2$ are the main absorbers in living tissues in the 764 to $824 \mathrm{~nm}$ wavelength range and that the light can penetrate the sample volume. $[\mathrm{Hb}]$ and $\left[\mathrm{HbO}_{2}\right]$ are estimated using the linear least-squares of multiwavelength $P A_{\lambda}$ values and the model in Eq. (1). $\mathrm{SO}_{2}$ and $\mathrm{HbT}$ are calculated from the following equations:

$$
\begin{aligned}
\mathrm{HbT} & =\left[\mathrm{HbO}_{2}\right]+[\mathrm{Hb}] \\
\mathrm{SO}_{2} & =\frac{\left[\mathrm{HbO}_{2}\right]}{\left[\mathrm{HbO}_{2}\right]+[\mathrm{Hb}]}
\end{aligned}
$$

In the case of brain imaging, the total hemoglobin concentration $(\mathrm{HbT})$ of the image can be interpreted as the cerebral blood volume (CBV) distribution.

\subsection{In-vitro blood test}

A functional PAT system was previously applied to measuring the $\mathrm{SO}_{2}$ levels of bovine blood samples in vitro. A blood sample was collected from a bovine subject, and high and low $\mathrm{SO}_{2}$ levels of blood were prepared by exposing the blood to $\mathrm{O}_{2}$ and $\mathrm{CO}_{2}$, respectively. These two blood samples were mixed with different volume ratios to achieve various $\mathrm{SO}_{2}$ levels (from $29 \%$ to $93 \%$ ), which were measured by a NIR spectrophotometer after collecting small samples and hemorrhaging the red blood cells using saporin. ${ }^{10}$ Mixed blood samples were injected into $0.25 \mathrm{~mm}$ inner-diameter optically and acoustically transparent tubes and varied inside the chicken breast tissue phantoms (thickness $=2 \mathrm{~mm}$ ). Three tubes were placed in the same plane of the phantom and separated by around $2 \mathrm{~mm}$. Three tubes with different $\mathrm{SO}_{2}$ level blood were imaged at the same time. Compared to the spectrophotometer results, the measured $\mathrm{SO}_{2}$ levels from the functional PAT images were linear to the optical measurements with a high linear regression coefficient, $\mathrm{R}=$ 0.99. However, there was a systematic bias in the measurement results because of overestimation of the Hb. Calibration results from previous the in-vitro blood tests were therefore applied. 


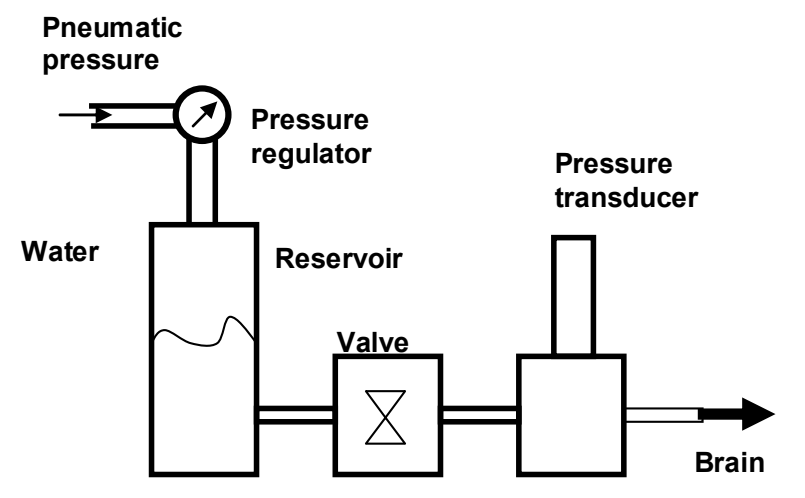

(a)

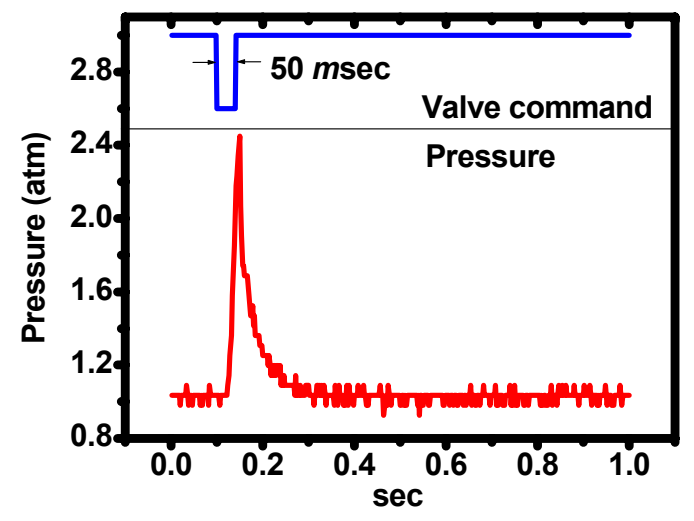

(b)

Figure 2. (a) Schematic representation of the experimental setup to produce fluid percussion injury in the rat, (b) the cortical impact pressure profile and value command

\subsection{Fluid percussion trauma}

Traumatic lesions of the rat brain were induced using a modification of the lateral fluid percussion procedure described by S. Toulmond et al. ${ }^{11}$ Sprague-Dawley rats weighing about 200 300 grams were used for the experiments. Surgery was performed under isoflurane gas aesthesia with a dose of $1 \%$ of pure oxygen at a $1 \mathrm{~L} / \mathrm{min}$ flow rate. A $2 \mathrm{~mm}$ diameter craniotomy was made at the level of the right parietal cortex; the dura was left intact. A $1 \mathrm{~mm}$ inner diameter Teflon tube was placed over the dura, anchored to the skull with dental cement and connected to the PC- commanded solenoid valve. The opposite side of the valve was connected to a sterile water chamber pressurized by a pneumatic supply of $5 \mathrm{~atm}$. A fluid percussion injury of the brain was induced by briefly opening the solenoid valve. The applied cortical pressure was measured by the pressure transducer (PX219, Omega) and recorded by an oscilloscope. Figure. 2 shows the fluid percussion device and the measured pressure on the cortex with the corresponding valve opening command for this experiment.

Imaging was performed under isoflurane gas aesthesia with the same dose as used in the surgery. The body temperature of the animal was maintained by controlling the temperature of the water heating pad at $36^{\circ} \mathrm{C}$. The pulse rate and the global arterial blood oxygenation were monitored during the entire experiment using a pulse oximeter (Model 8600, Nonin Medical Inc.) After imaging, the mice were sacrificed using pentobarbital (120 mg/kg, IP). The imaged brains were excised and stained with $\mathrm{H} \& \mathrm{E}$ for histology. All of the experimental animal procedures were approved by the University Laboratory Animal Care Committee of Texas A\&M University.

\section{RESULTS AND DISCUSSIONS}

Time course functional photoacoustic images of a rat brain with TBI and a corresponding open-skull photo are shown in Figure 3. The photo of the brain in Fig.3(a) indicates a hemorrhagic episode near the site of injury. Two of the HbT images of the rat brain are presented in Figures 3(b) and (c), where the color bars are identical. Compared to the brain image taken before the TBI (Figure 3(b)), the image acquired $\sim 0.5$ hour after the TBI (Figure 3(c)) shows increased blood content at the vicinity of the impact site, where the hemorrhage on the brain cortex is observed in Figure 3(a). The differential image in Figure 3(d) is the result of subtraction of the pre-trauma image in Figure 4(b) from the post trauma image in Figure 3(c). This image depicts the distribution of cerebral blood volume (CBV) changes caused by the

trauma. It is noted that there are two distinctive changes. One is the increased HbT near the impact zone, and the other is a substantial decrease (20\%) in blood volume in the median fissure (MF) after the TBI. The alteration in oxy- 


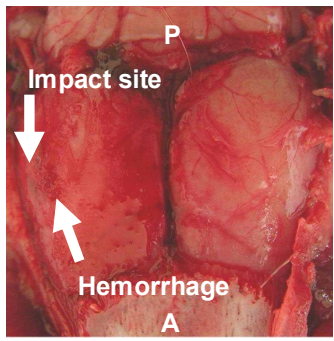

(a)

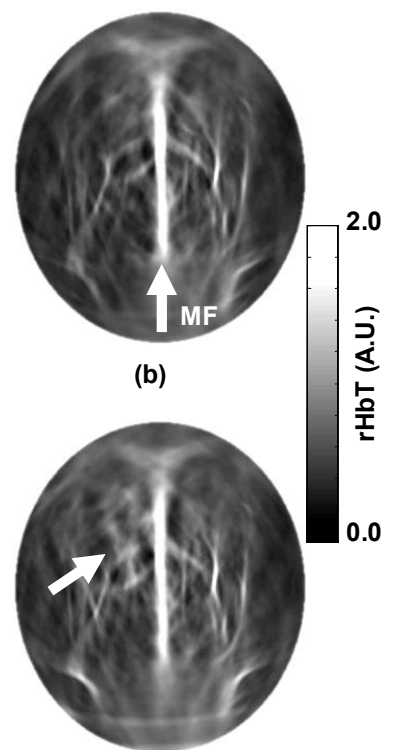

(c)

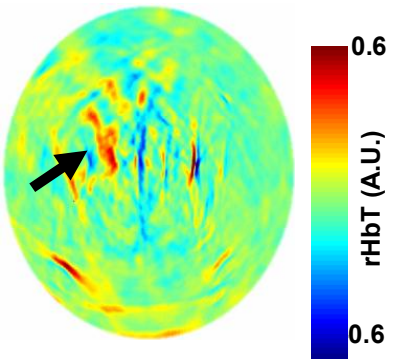

(d)

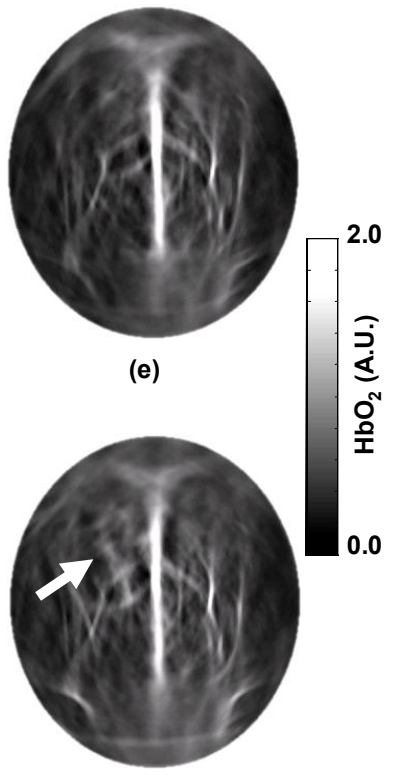

(f)

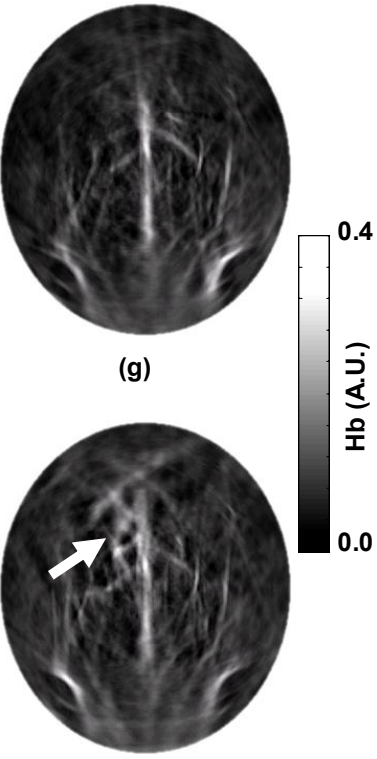

(h)

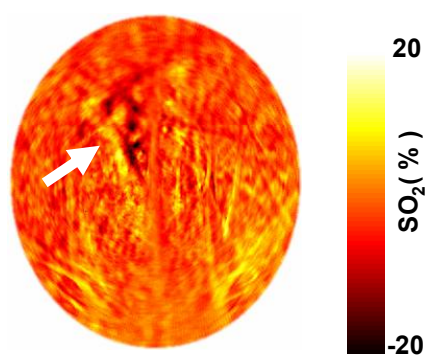

(i)

Figure 3 In-vivo functional photoacoustic images of the brain prior to, and following, fluid-percussion brain injury. (a) Open-skull photograph of the brain cortex obtained after the image acquisition for functional PAT. A: anterior side of the brain, P: posterior side. (b),(c) HbT image acquired before and $\sim 30 \mathrm{~min}$ after the brain injury, respectively. (d) Differential HbT image obtained by subtracting the pre-TBI image from the post-TBI image (image $\mathrm{d}=$ image $\mathrm{c}$ - image b). (e), (f) Oxyhemoglobin $\left(\mathrm{HbO}_{2}\right)$ image acquired before and $\sim 30$ min after the brain injury, respectively. (g), (h) Deoxyhemoglobin $\left(\mathrm{HbO}_{2}\right)$ image acquired before and $\sim 30 \mathrm{~min}$ after the brain injury, respectively. (i) Differential $\mathrm{SO}_{2}$ image obtained by subtracting the pre-TBI $\mathrm{SO}_{2}$ image from the post-TBI $\mathrm{SO}_{2}$ image. The arrows indicate the injury site. MF: median fissure.

hemoglobin and deoxy-hemoglobin are are shown in Figure 3(e), (f), (g), and (h). The post-trauma image of deoxyhemoglobin shows substantial changes near the hemorrhage site. The differential image in Figure 3(i) is the result of the subtraction of the pre-trauma $\mathrm{SO}_{2}$ image based on Figure 3(e) and (g) from the post trauma $\mathrm{SO}_{2}$ image calculated from Figure 3(f) and (h). It indicates that the both an increase and a decrease of $\mathrm{SO}_{2}$ at the impact site, where the strong $\mathrm{HbT}$ values are detected. It is noted that there are no substantial changes except in the hemorrhage zone. The $\mathrm{SO}_{2}$ value of the small vasculature on the cortex seems not to be affected by the TBI in this experiment.

In addition to imaging the entire brain, one specific position of the brain was monitored using a photoacoustic signal every 1 min to pick up the transient hemodynamics caused by the TBI. In this research, the median fissure (MF) was chosen to detect fast changing physiological values.. Figure 4(a) depicts a representative photoacoustic signal from the MF when the transducer was located so that its axis was perpendicular to the MF. Figure 4(b) shows the time course $\mathrm{HbT}$ and $\mathrm{SO}_{2}$ levels at the MF. During the pre-TBI period, both values are stable. In the post-TBI period, the cerebral 


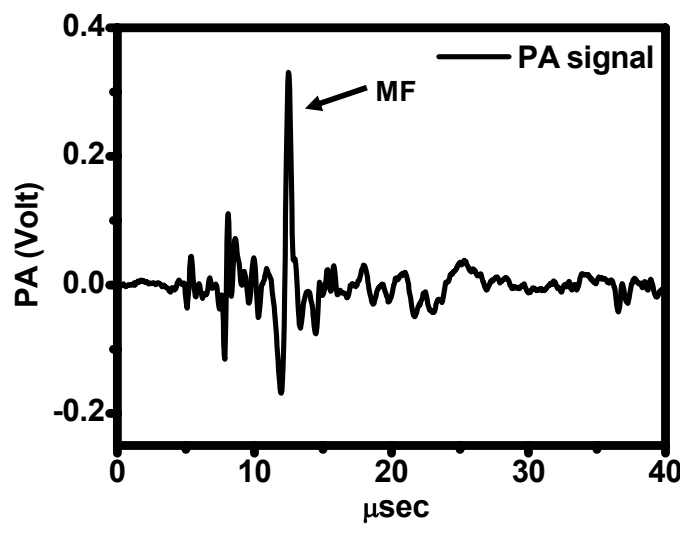

(a)

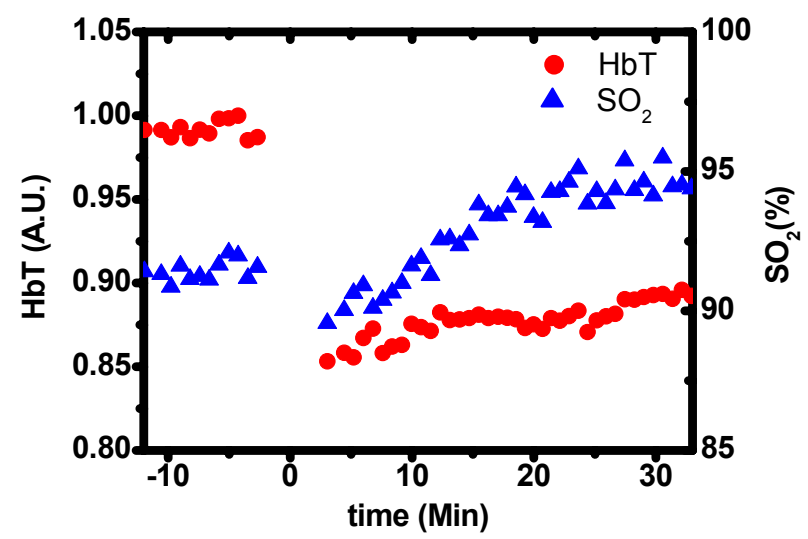

(b)

Figure 4 Total hemoglobin concentration $(\mathrm{HbT})$ and oxygen saturation level $\left(\mathrm{SO}_{2}\right)$ in the median fissure (MF) of the brain prior to, and following, the fluid-percussion brain injury. (a) Representative photoacoustic signal coming from the MF. (b) Transient $\mathrm{HbT}$ and $\mathrm{SO}_{2}$ in the MF prior to, and following, the fluid-percussion brain injury. Dotted line in (b) indicates the initiation of the brain injury.

blood volume measured from the $\mathrm{HbT}$ drops $20 \%$ immediately and remains the same for 30 minutes, at which time we finished the measurement. On the other hand, the oxygen saturation gradually increases from 91 to $95 \%$.

Studies of TBI imaging should expand our understanding of the hemodynamics underlying traumatic episodes and help in the development of treatments. In this study, we were interested in monitoring TBI in rats by acquiring the hemoglobin concentration and the hemoglobin oxygenation in the cortex of the brain non-invasively through the intact skin and skull. We described the functional photoacoustic tomography system used to measure these parameters and provided proof-of-principle experiments to demonstrate the system for TBI imaging. Figure 3 demonstrates the method's capability to visualize brain hemodynamics under traumatic injury, which is inaccessible to the conventional optical oxygen saturation imaging modalities. Higher blood volume was observed at the injury site, which was identified by the open-skull image. The increased $\mathrm{HbT}$ level was also not an unexpected result. Focal injury, caused by the fluid percussion model, usually accompanies hemorrhaging and hematoma formation, both of which involve increases in blood volume that result from breakages in the vasculature. ${ }^{2}$ The heterogeneous $\mathrm{SO}_{2}$ change at the injury site may result from the same source. The imaging results indicate that the fPAT can be an effective modality for locating and evaluating brain injury. In addition to imaging, the single point hemodynamic results in Figure 4 provide information on overall brain metabolism alterations after a TBI. First, the sudden decrease in HbT at the MF implies that the blood supply is reduced either by a hypotensive episode following regulatory impairment or by an increase in intracranial pressure, which is commonly observed in the TBI patient. Secondly,the increased $\mathrm{SO}_{2}$ at the MF, the major venous vasculature inside brain, implies reduced brain activity, which is proportional to the $\mathrm{SO}_{2}$ difference in the arterial and venous blood vessels. Throughout the experiment, the $\mathrm{SO}_{2}$ in the arterial blood vessels was continuously monitored by a pulse oxymeter. Changes were not noticeable even under TBI, but the venous MF deveoped a higher $\mathrm{SO}_{2}$. The $\mathrm{HbT}$ and $\mathrm{SO}_{2}$ values of pre- and post TBI in Figure 4 can be utilized to calculate the cerebral metabolic rate of oxygen (CMRO2) that is commonly used to quantify this brain activity. ${ }^{12}$ Compared with the CMRO2 value of pre TBI, it dropped more than $40 \%$ after the injury. In the near future, we intend to design a set of experiments that involves conventional invasive tools to measure and verify this value.

\section{Conclusion}

In this study, rats with a traumatic brain injury (TBI) were imaged with functional photoacoustic tomography. In-vivo and non-invasive high resolution brain cortex vasculature images of oxygen saturation and total hemoglobin concentration were produced that visualize the hemodynamic changes after the TBI. Trauma induced hematomas and 
blood oxygen saturation level changes were detected. Hemodynamics information was extracted from the images and from single point measurements, and the trends were consistent with the known physiological responses to TBI. Because of the location of the injury site inside the brain, conventional optical imaging modalities have not yet been able to detect in vivo homodynamic information with sub $\mathrm{mm}$ spatial resolution. The demonstrated functional photoacoustic tomography technique will be useful for future studies on TBI related metabolic changes and neuroprotective therapies.

\section{Acknowledgment}

This research was supported in part by the National Institutes of Health grants R01 EB000712 and R01 NS46214. J.T. Oh was supported in part by the Post-doctoral Fellowship Program of Korea Science \& Engineering Foundation (KOSEF).

\section{References}

1. H. Laurer, P. Lenzlinger, T. McIntosh, “ Models of traumatic brain injury”, European J. Trauma 3, pp. 95-110 (2000).

2. T. Gennarelli, “Animate models of human head injury”, J. Neurotrauma 11, pp. 397-406 (1994).

3. http://www.trauma.org/neuro/neuromonitor.html

4. H, Mao, and G. Berns, "MRI in the study of brain function: clinical perspectives," Medicamundi 46, pp. 28-38 (2002).

5. B. Albensi, S. Knoblach, B. Chew, M. O’Reilly, A. Faden, and J. Pekar, "Diffusion and high resolution MRI of traumatic brain injury in rats: time course and correlation with histology," Exp. Neurology 162, pp. 61-72 (2000).

6. C. Cheung, J.P. Culver, K. Takahashi, J.H. Greenberg ,A.G. Yodh, In vivo cerebrovascular measurement combining diffuse near-infrared absorption and correlation spectroscopies, Phys. Med. Biol. 46, pp. 2053-2065 (2001).

7. X. Wang, Y. Pang, G. Ku, X. Xie, G. Stoica, and L. Wang, "Noninvasive laser-induced photoacoustic tomography for structural and functional in vivo imaging of the brain," Nat, Biotechnol. 21, pp. 803-806 (2003).

8. X. Wang, X. Xie, G. Ku, G. Stoica, and L. Wang, "Functional photoacoustic tomography for non-invasive imaging of cerebral blood oxygenation and blood volume in rat brain in vivo," SPIE 5697, pp. 1-6 (2005).

9. M. Xu, Y. Xu, and L. Wang, "Time-domain reconstruction algorithms and numerical simulations for thermoacoustic tomography in various geometries," IEEE Trans. on Biomed. Eng. 50, pp. 1086-1099 (2003).

10. P. Scheid, and M. Meyer, "Mixing technique for study of oxygen-haemoglobin equilibrium: a critical evaluation," $J$. Appl. Physiol. 45, pp. 818-822 (1978).

11. S. Toulmond, D. Duval, A. Serrano, B. Scatton and J. Benavides, "Biochemical and histological alterations induced by fluid percussion brain injury in the rat," Brain Res. 620, pp. 24-31 (1993).

12. J. Cruz, "The first decade of continuous monitoring of jugular bulb oxyhemoglobin saturation: Management strategies and clinical outcome," Critical Care Medicine. 26, pp. 344-351 (1998) . 